\title{
Prognostic significance of mucinous component in gastric adenocarcinoma after radical D2 gastrectomy
}

This article was published in the following Dove Press journal: OncoTargets and Therapy

Lei Cai'

Yan $\mathrm{Li}^{2}$

Xue-wen Yang'

Xiao Lian'

Man Guo'

Shu-ao Xiao'

Wen-bin Wang'

Hong-wei Zhang'

'Division of Digestive Surgery, Xijing Hospital of Digestive Diseases, Fourth Military Medical University, 2Department of Anesthesiology, Northwest Women's and Children's Hospital, Xi'an, China
Correspondence: Hong-wei Zhang Division of Digestive Surgery, Xijing Hospital of Digestive Diseases, Fourth Military Medical University, 127 West Changle Road, Xi'an,

Shaanxi 710032, China

Tel +8613319261910

Fax +86298253 9041

Email zhanghw153I@163.com
Background: The mucinous component is a special histologic factor in gastric adenocarcinoma. The aim of this study was to assess the prognostic significance of mucinous component in gastric adenocarcinoma according to proportion.

Patients and methods: Candidate patients with gastric adenocarcinoma were given radical D2 gastrectomies from September 2008 to May 2015 in our division. Clinicopathologic data and prognosis were monitored and analyzed among gastric adenocarcinoma patients with various proportions of mucinous component.

Results: A total of 690 gastric adenocarcinomas with various proportions of mucinous component from 6,025 gastric adenocarcinoma patients were included. Higher numbers of patients with mucinous component came from: young patients, females, those with drinking history, at lower locations, Borrmann type III and IV, T4 stage, and positive for dissected lymph nodes. Tumors and pathological molecular markers showed more positivity in CEA, CA19-9, S100, and CD34. As the various proportions increased, more mucinous component seemed to be accompanied by more Borrmann type III and IV, T4 stage, and more positive expression of CEA and CA19-9. However, no significant difference in 5-year overall survival rate was observed among various proportions or existence of mucinous component. Also, proportion or existence of mucinous component was not an independent prognostic factor in multivariate analysis.

Conclusion: Mucinous component was not a prognostic factor for gastric adenocarcinoma after radical D2 gastrectomy, no matter what proportion the component comprised. However, gastric adenocarcinoma with mucinous component showed specific clinicopathological characteristics, such as more advanced tumor stage, different age and sex, and more positive rate of molecular markers, which might provide a new strategy for optimal individual diagnosis and therapies.

Keywords: mucinous component, mucinous gastric carcinoma, gastric adenocarcinoma with mucinous component, pure gastric adenocarcinoma, radical D2 gastrectomy, prognosis

\section{Introduction}

Mucinous gastric carcinoma (MGC) is considered a special histologic type of gastric adenocarcinoma with a substantial amount of extracellular mucin $(\geq 50 \%$ of tumor volume) within tumor areas defined by the World Health Organization (WHO)., ${ }^{1,2}$ Recently, some studies focussed on the MGC. ${ }^{3-5}$ Tang et $\mathrm{al}^{6}$ found that MGC was an aggressive malignancy with unique clinicopathological features in $244 \mathrm{MGC}$ patients who underwent radical gastrectomy with D2 lymphadenectomy. In Isobe et al's study, 70 MGC patients were compared with 2,492 non-MGC (NMGC) patients, and although MGC is rarer and mostly detected at an advanced stage, the diagnosis of 
the mucinous histological subtype was not an independent prognostic factor. Zhao et al's results showed MGC was more likely to have large tumors and more advanced tumor stage (II and III) than NMGC, and the thicker gastric wall, layered enhancement pattern, and calcification were highly suggestive computed tomography features for differentiating MGC from NMGC. ${ }^{8}$ It is been stated that this subtype of gastric adenocarcinoma seems to be accompanied by advanced tumor stage and poor prognosis, ${ }^{9}$ but the molecular markers in this histologic type remain unknown.

In 2014, Park et al reported on the prognostic significance of neuroendocrine components in GC, finding that different proportions of neuroendocrine components might indicate different prognoses. ${ }^{10}$ Also, the mucinous component has been observed in gastric adenocarcinoma frequently, ${ }^{11,12}$ but the biological characteristics and prognosis of mucinous component in gastric adenocarcinoma after radical D2 gastrectomy are not yet clear. In this study, we explored the clinicopathologic (especially molecular tumor and pathology markers) and prognostic significance of mucinous component in gastric adenocarcinoma after radical D2 gastrectomy according to proportion.

\section{Patients and methods Selection of patients}

Candidate patients who had undergone radical D2 gastrectomy for gastric adenocarcinoma in the Division of Digestive Surgery of Xijing Digestive Hospital between September 2008 and May 2015 were enrolled. This retrospective study adhered to the guidelines established by the Declaration of Helsinki and was approved by the institutional review board of Xijing Hospital. Signed informed consent forms were received from all enrolled patients. All patients were evaluated carefully for anesthesia and operation safety. Patients had undergone radical D2 gastrectomy as the standard approach, and pathologic diagnosis was done by at least two trained and experienced gastrointestinal pathologists using the WHO criteria. ${ }^{13-15}$

\section{Data collection}

Clinicopathologic characters for each patient - age, sex, history of drinking and smoking, tumor location, Borrmann type, tumor biomarkers (AFP, CEA, CA125, CA19-9), molecular pathological markers (S100, EGFR, HER2) - were obtained from surgical records, medical charts, and postoperative histopathology reports. We collected data on depth of invasion and lymph-node metastasis instead of pathological stage, in order to assess the significance of different $\mathrm{T}$ and $\mathrm{N}$ stages on prognosis separately.

\section{Prognosis and statistical analysis}

Gastric adenocarcinoma patients with or without mucinous component were selected based on $\mathrm{T}$ and $\mathrm{N}$ stage, tumor location, and Borrmann type, while patients with various proportions of mucinous component were accessed based on T stage and Borrmann type, in order to reduce differences in clinicopathological characteristics, using Gmatch methods.

Selected patients were followed up according to standard protocol (3-month interval for the first 2 years, every 6 months for the subsequent 3 years, and yearly thereafter) for 1-67 months. The 5-year overall survival rate was estimated by Kaplan-Meier method and compared by log-rank test. The Cox proportional hazard model was employed for multivariate analysis. All statistical analyses were performed with SPSS version 19.0. $P$-values $<0.05$ were considered statistically significant, and all tests were two-sided.

\section{Results \\ Patient features}

A total of 6,205 patients with gastric cancer were admitted to our division from September 2008 to May 2015, and 3,537 adenocarcinoma patients were enrolled, including $176 \mathrm{MGC}$ ( $\geq 50 \%$ of extracellular mucin within tumors areas), 514 gastric adenocarcinoma with mucinous component (GAM; 0-50\% extracellular mucin within tumor areas), and 2,847 pure gastric adenocarcinoma (PGA) without mucinous component (without extracellular mucin within tumor areas).

\section{Comparison between gastric adenocarcinoma with and without mucinous component}

GAM and MGC patients were mostly female, younger ( $<60$ years), with drinking history, lower tumor location, Borrmann types III and IV, T4 stage, and more lymph-node metastasis (Table 1). Analysis of molecular markers showed more positive expression of CEA, CA19-9, and S100 and less positive expression of AFP and HER2 $(>++$ and fluorescence in situ hybridization) in GAM and MGC than in PGA (Table 1). By Gmatch, 646 pairs were obtained and assessed, with the overall rate of visits available for analysis at $96.5 \%$. The 5-year overall survival rate showed no significant difference between patients with or without mucinous component after radical D2 gastrectomy, even with different $\mathrm{T}$ stages (Figure 1). Ten factors assessed in univariate analysis had significant influence on long-term survival (Table 2). Multivariate analysis indicated $\mathrm{T}$ stage, $\mathrm{N}$ stage, and CEA were independent prognostic factors, while existence of mucinous component in gastric adenocarcinoma was not a prognostic factor for patients after radical D2 gastrectomy (Table 2). 
Table I Clinicopathological characteristics of patients with/ without mucinous component

\begin{tabular}{|c|c|c|c|}
\hline Variables & $\begin{array}{l}\text { With, } \\
n=690\end{array}$ & $\begin{array}{l}\text { Without, } \\
n=2,847\end{array}$ & $P$-value \\
\hline Age, years & & & 0.018 \\
\hline$\leq 30$ & $12(1.7 \%)$ & 32 (I.I\%) & \\
\hline $31-40$ & $47(6.8 \%)$ & $153(5.4 \%)$ & \\
\hline $4 I-50$ & $136(19.7 \%)$ & $524(18.4 \%)$ & \\
\hline $51-60$ & $25 \mathrm{I}(36.4 \%)$ & $913(32.1 \%)$ & \\
\hline $6 I-70$ & 173 (25.1\%) & $857(30.1 \%)$ & \\
\hline $7 I-80$ & $67(9.7 \%)$ & $350(12.3 \%)$ & \\
\hline$>80$ & $4(0.6 \%)$ & $18(0.6 \%)$ & \\
\hline Sex & & & 0.009 \\
\hline Male & $512(74.2 \%)$ & 2,244 (78.8\%) & \\
\hline Female & $178(25.8 \%)$ & 603 (2I.2\%) & \\
\hline Smoking history & & & 0.083 \\
\hline Yes & $594(86.1 \%)$ & $2,374(83.4 \%)$ & \\
\hline No & $96(13.9 \%)$ & $473(16.6 \%)$ & \\
\hline Drinking history & & & 0.000 \\
\hline Yes & 209 (30.3\%) & 342 (I2\%) & \\
\hline No & 481 (69.7\%) & $\mathrm{I}, 505(88 \%)$ & \\
\hline Location & & & 0.035 \\
\hline Upper & $211(30.6 \%)$ & $998(35.1 \%)$ & \\
\hline Middle & $118(17.1 \%)$ & $513(18 \%)$ & \\
\hline Lower & $345(50 \%)$ & $\mathrm{I}, 295(45.5 \%)$ & \\
\hline Whole & $16(2.3 \%)$ & $4 \mathrm{I}(\mathrm{I} .4 \%)$ & \\
\hline Macroscopic type & & & 0.001 \\
\hline Early gastric cancer & 87 (I3.3\%) & $518(19 \%)$ & \\
\hline Borrmann I & 47 (7.2\%) & 207 (7.6\%) & \\
\hline Borrmann II & $153(23.3 \%)$ & $686(25.2 \%)$ & \\
\hline Borrmann III & 290 (44.2\%) & $1,070(39.3 \%)$ & \\
\hline Borrmann IV & $79(12 \%)$ & $240(8.8 \%)$ & \\
\hline Depth on invasion & & & 0.000 \\
\hline $\mathrm{TI}$ & 89 (I2.9\%) & $550(19.1 \%)$ & \\
\hline T2 & $84(12.2 \%)$ & $451(16 \%)$ & \\
\hline T3 & $240(34.8 \%)$ & $1,030(36.2 \%)$ & \\
\hline $\mathrm{T} 4$ & $276(40.1 \%)$ & $816(28.7 \%)$ & \\
\hline \multicolumn{2}{|c|}{ Lymph-node metastasis } & & 0.000 \\
\hline No & $182(26.5 \%)$ & $\mathrm{I}, 057(37.3 \%)$ & \\
\hline NI & $176(25.6 \%)$ & $551(19.5 \%)$ & \\
\hline N2 & $104(15.1 \%)$ & 491 (17.4\%) & \\
\hline N3 & $225(32.8 \%)$ & $727(27.1 \%)$ & \\
\hline CEA & & & 0.000 \\
\hline Positive & $185(30 \%)$ & $450(17.6 \%)$ & \\
\hline Negative & $432(70 \%)$ & $2,106(82.4 \%)$ & \\
\hline AFP & & & 0.003 \\
\hline Positive & $18(3 \%)$ & $15 \mid(6.1 \%)$ & \\
\hline Negative & $583(97 \%)$ & $2,312(93.9 \%)$ & \\
\hline CA19-9 & & & 0.000 \\
\hline Positive & 178 (29.3\%) & 446 (I7.9\%) & \\
\hline Negative & 429 (70.7\%) & $2,048(82.1 \%)$ & \\
\hline CAI 25 & & & 0.775 \\
\hline Positive & $31(5.2 \%)$ & $116(4.8 \%)$ & \\
\hline Negative & 557 (94.7\%) & $2,212(95.2 \%)$ & \\
\hline S100 & & & 0.000 \\
\hline Positive & $432(87.4 \%)$ & I,585 (76.7\%) & \\
\hline Negative & $62(12.6 \%)$ & $48 \mathrm{I}(23.3 \%)$ & \\
\hline EGFR & & & 0.992 \\
\hline Positive & $324(61.5 \%)$ & $\mathrm{I}, 342$ (6I.5\%) & \\
\hline Negative & $203(38.5 \%)$ & $840(38.5 \%)$ & \\
\hline HER2 & & & 0.000 \\
\hline$>++$ & 219 (4I.4\%) & $\mathrm{I}, \mathrm{I} 09$ (50.8\%) & \\
\hline$\leq++$ & $310(58.6 \%)$ & I,075 (49.2\%) & \\
\hline
\end{tabular}

Comparison among various proportions of mucinous component

Compared with GAM, MGC occurred more frequently in Borrmann type III and IV, with more T4 stage (Table 1). As for molecular markers, positive expression of CA19-9 in MGC was higher than that of $\operatorname{GAM}(P<0.05)$, while the positive rate of EGFR and HER2 ( $>++$ and fluorescence in situ hybridization) in MGC was less than that of $\operatorname{GAM}(P<0.05)$ (Table 3). With the same approach by Gmatch, 155 pairs were selected, with overall visits available for analysis also at $96.5 \%$. MGC and GAM had similar 5-year overall survival rates $(P>0.05)$ (Figure 2$)$. When compared with prognosis by T stage, data showed no significant differences $(P>0.05)$ (Figure 2). Eight factors evaluated in univariate analysis had a significant influence on long-term survival (Table 3). Multivariate analysis indicated that independent prognostic factors were $\mathrm{N}$ stage and CEA, and proportion of mucinous component in gastric adenocarcinoma was not a prognostic factor for patients after radical D2 gastrectomy (Table 4).

\section{Discussion}

Gastric adenocarcinoma is one of the most common cancers in developing countries, especially in China. Because of its heterogeneity, therapeutic effectiveness is not satisfactory, which might be due to different proportions of histologic component. MGC is considered a special subtype of gastric adenocarcinoma, and MGC characteristics have been reported by several studies as deeper invasion, lower location, more lymph-node metastasis, and poor prognosis. ${ }^{2,6,9,16,17}$ In Zu et al's study, MGC survival was correlated with stage at diagnosis and mucinous histologic type was an independent prognostic factor. ${ }^{16}$ Yin et al compared $196 \mathrm{MGC}$ with 2,573 PGA samples, which showed MGC was more advanced at the time of diagnosis and received less curative gastrostomy, but the prognosis of MGC was not significantly different from PGA after curative gastrostomy. ${ }^{2}$ For patients in our division, MGC is a rare histologic type in gastric adenocarcinoma and diagnosed mostly in an advanced stage, but is not a prognostic significance after radical D2 gastrectomy. ${ }^{9}$

Lots of studies have reported on the clinical-pathological characteristics and prognosis of MGC, $4,6,7,18$ but few have focused on the prognostic significance of mucinous component in gastric adenocarcinoma, even though the coexistence of mucinous component and gastric adenocarcinoma is observed frequently. Kim et a ${ }^{19}$ revealed there were some differences in several clinicopathological features of signet-ring cell, mucinous (MGC), papillary, and lymphoepitheliomalike subtypes of advanced gastric cancer, and no statistical differences in survival were found among these after 

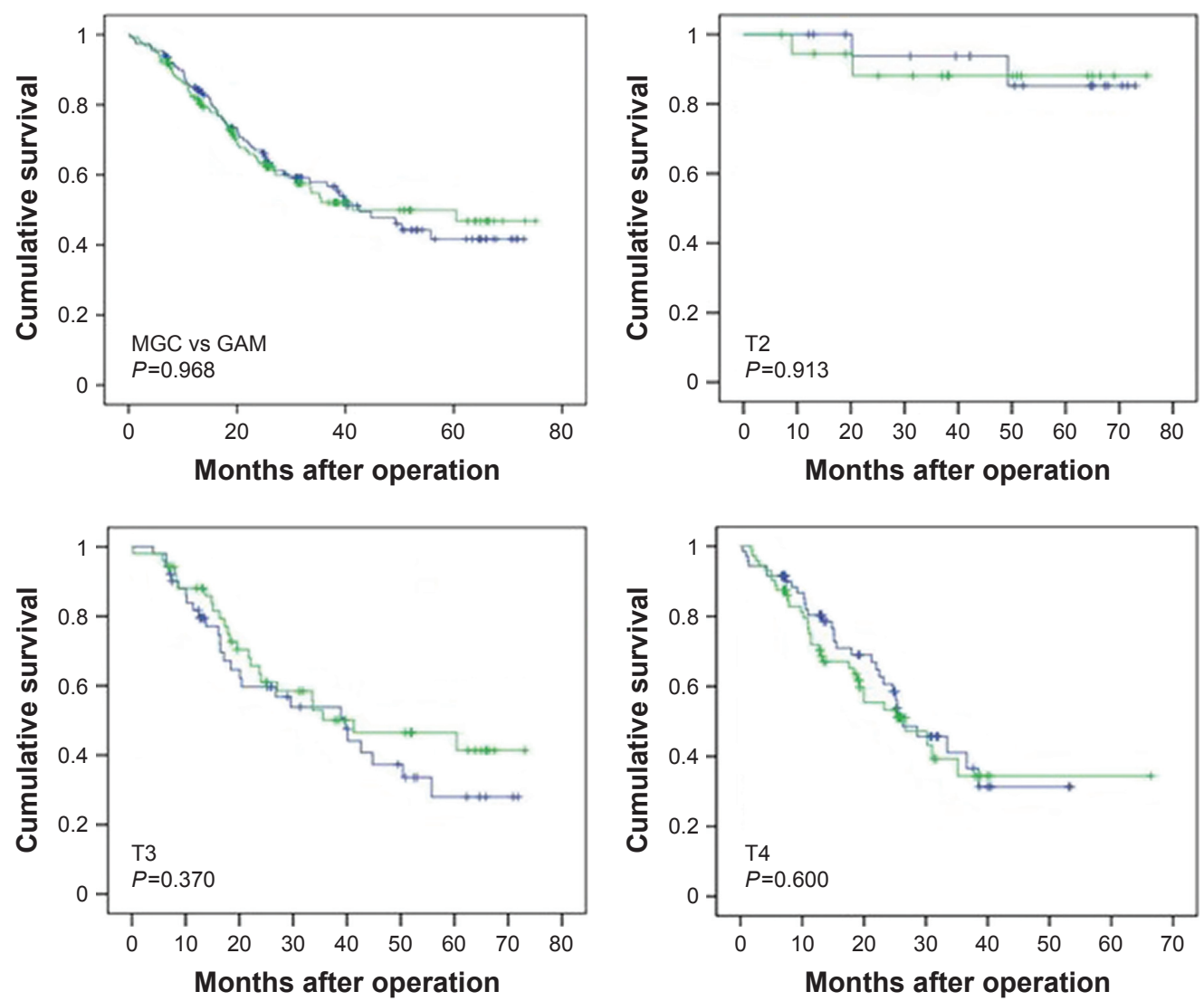

$$
\begin{aligned}
& \text { Group } \\
& -\mathrm{MGC}+\mathrm{GAM}+\mathrm{MGC} \text {-censored }+ \text { GAM-censored }
\end{aligned}
$$

Figure I Patients with or without mucinous component had no difference in prognosis after D2 radical gastrectomy.

Abbreviations: MGC, mucinous gastric carcinoma; GAM, gastric adenocarcinoma with mucinous component.

R0 gastrectomy. In another study, ${ }^{17}$ clinical and pathological features and prognoses of GMC and gastric signet-ring cell types differed. Therefore, the treatment of the two diseases should be individualized.

Table 2 Univariate and multivariate analyses of prognostic factors with/without mucinous component after radical D2 gastrectomy

\begin{tabular}{llll}
\hline Variables & $\beta$ & HR (95\% CI) & P-value \\
\hline Univariate & & & \\
T stage & 0.79 & $2.203(1.948-2.492)$ & 0.000 \\
N stage & 0.673 & $1.96(1.792-2.143)$ & 0.000 \\
Age & 0.098 & $1.103(1.021-1.192)$ & 0.013 \\
Location & -0.125 & $0.882(0.799-0.974)$ & 0.013 \\
Borrmann type & -0.13 & $0.878(0.832-0.926)$ & 0.019 \\
CEA & 0.735 & $2.086(1.702-2.557)$ & 0.000 \\
AFP & 0.734 & $2.084(1.447-3.002)$ & 0.000 \\
CAI9-9 & 0.675 & $1.963(1.607-2.398)$ & 0.000 \\
CAI25 & 1.262 & $3.531(2.557-4.875)$ & 0.000 \\
SI00 & 1.165 & $3.206(2.16-4.785)$ & 0.000 \\
Multivariate & & & \\
T stage & 0.438 & $1.549(1.258-1.908)$ & 0.000 \\
N stage & 0.52 & $1.682(1.483-1.907)$ & 0.000 \\
CEA & 0.345 & $1.412(1.097-1.817)$ & 0.007 \\
\hline
\end{tabular}

Table 3 Higher proportions of mucinous component correlated with more advanced tumor stage and positive CEA, CA19-9, EGFR, and HER2*

\begin{tabular}{llll}
\hline Variables & $\begin{array}{l}\text { MGC, } \\
\mathbf{n}=\mathbf{I 7 6}\end{array}$ & $\begin{array}{l}\text { GAM, } \\
\mathbf{n}=\mathbf{5} \text { I4 }\end{array}$ & P-value \\
\hline Age, years & $2(1.1 \%)$ & $10(1.9 \%)$ & 0.624 \\
$\leq 30$ & $7(2.7 \%)$ & $40(7.8 \%)$ & \\
$31-40$ & $33(18.8 \%)$ & $103(20 \%)$ & \\
$4 I-50$ & $66(37.5 \%)$ & $185(36 \%)$ & \\
$5 I-60$ & $47(26.7 \%)$ & $126(24.5 \%)$ & \\
$6 I-70$ & $20(11.4 \%)$ & $47(9.1 \%)$ & \\
$7 I-80$ & $1(0.6 \%)$ & $3(0.6 \%)$ & 0.139 \\
$>80$ & & & \\
Sex & $138(78.4 \%)$ & $374(72.8 \%)$ & \\
Male & $38(21.6 \%)$ & $140(27.2 \%)$ & 0.897 \\
Female & & & \\
Smoking history & $15 I(85.8 \%)$ & $443(86.2 \%)$ & \\
Yes & $25(14.2 \%)$ & $71(13.8 \%)$ & \\
No & & & \\
Drinking history & $150(85.2 \%)$ & $59(11.5 \%)$ & \\
Yes & $26(14.8 \%)$ & $455(88.5 \%)$ & \\
No & & & \\
Location & $6 I(34.7 \%)$ & $150(29.2 \%)$ & \\
Upper & $25(14.2 \%)$ & $93(18.1 \%)$ & \\
Middle & $84(47.7 \%)$ & $26 \mid(50.8 \%)$ & \\
Lower & $6(3.4 \%)$ & $10(1.9 \%)$ & \\
Whole & & & $($ Continued $)$ \\
\hline & & &
\end{tabular}


Table 3 (Continued)

\begin{tabular}{|c|c|c|c|}
\hline Variables & $\begin{array}{l}\text { MGC, } \\
n=176\end{array}$ & $\begin{array}{l}\text { GAM, } \\
n=5 \text { I4 }\end{array}$ & $P$-value \\
\hline \multicolumn{3}{|l|}{ Macroscopic type } & 0.008 \\
\hline Early gastric cancer & $13(8.2 \%)$ & 74 (I4.9\%) & \\
\hline Borrmann I & $6(3.8 \%)$ & $41(8.2 \%)$ & \\
\hline Borrmann II & $32(20.1 \%)$ & $121(24.3 \%)$ & \\
\hline Borrmann III & $83(52.2 \%)$ & 207 (41.6\%) & \\
\hline Borrmann IV & 25 (I5.7\%) & $54(12.8 \%)$ & \\
\hline \multicolumn{3}{|l|}{ Depth on invasion } & 0.000 \\
\hline TI & $15(8.5 \%)$ & 74 (I4.4\%) & \\
\hline T2 & $20(11.4 \%)$ & 64 (12.5\%) & \\
\hline T3 & 55 (31.8\%) & $185(36 \%)$ & \\
\hline T4 & 85 (48.3\%) & $191(37.2 \%)$ & \\
\hline \multicolumn{3}{|c|}{ Lymph-node metastasis } & 0.000 \\
\hline No & $37(20.3 \%)$ & 145 (28.3\%) & \\
\hline NI & $82(46.6 \%)$ & 145 (I8.3\%) & \\
\hline N2 & $17(16.3 \%)$ & $87(17 \%)$ & \\
\hline N3 & $38(16.9 \%)$ & $187(36.5 \%)$ & \\
\hline \multicolumn{3}{|l|}{ CEA } & 0.04 \\
\hline Positive & $58(36.3 \%)$ & 127 (27.8\%) & \\
\hline Negative & $102(63.8 \%)$ & 330 (72.2\%) & \\
\hline \multicolumn{3}{|l|}{ AFP } & 0.137 \\
\hline Positive & $2(1.3 \%)$ & $16(3.6 \%)$ & \\
\hline Negative & $156(98.7 \%)$ & 427 (96.4\%) & \\
\hline \multicolumn{3}{|l|}{ CAI9-9 } & 0.001 \\
\hline Positive & $63(39.1 \%)$ & $115(25.8 \%)$ & \\
\hline Negative & 98 (60.9\%) & $33 \mathrm{I}(74.2 \%)$ & \\
\hline \multicolumn{3}{|l|}{ CAI 25} & 0.416 \\
\hline Positive & $10(6.5 \%)$ & 21 (4.8\%) & \\
\hline Negative & 143 (93.5\%) & $4 \mid 4$ (95.2\%) & \\
\hline \multicolumn{3}{|l|}{ S100 } & 0.067 \\
\hline Positive & 94 (82.5\%) & 338 (88.9\%) & \\
\hline Negative & 20 (17.5\%) & 42 (II.I\%) & \\
\hline \multicolumn{3}{|l|}{ EGFR } & 0.006 \\
\hline Positive & $6 \mathrm{I}(50.8 \%)$ & 263 (64.6\%) & \\
\hline Negative & 59 (49.2\%) & 144 (35.4\%) & \\
\hline \multicolumn{3}{|l|}{ HER2 } & 0.02 \\
\hline$>++$ & 39 (32.3\%) & 180 (44.1\%) & \\
\hline$\leq++$ & 82 (67.7\%) & 228 (55.9\%) & \\
\hline
\end{tabular}

Note: $*>++$ and FISH test-positive.

Abbreviations: MGC, mucinous gastric carcinoma; GAM, gastric adenocarcinoma with mucinous component; FISH, fluorescence in situ hybridization.

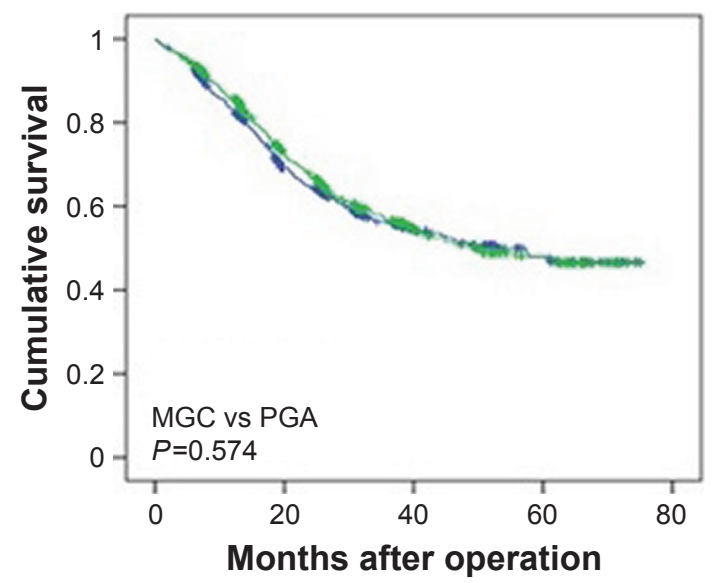

In 2014, Park et a $1^{10}$ explored the prognostic significance of neuroendocrine components in $\mathrm{GC}$ and found different proportions of neuroendocrine components led to different prognoses. In this study, we assessed differences among various proportions of mucinous component to explore the prognostic significance of mucinous component in gastric adenocarcinoma after radical D2 gastrectomy. We found that characteristics of gastric adenocarcinoma with the presence of mucinous component in patients of a more advanced stage, who were younger and female, with a drinking history, and in those with gastric adenocarcinoma in the lower stomach, even though it is said that the incidence of gastric adenocarcinoma in the upper stomach increases year by year. However, the two groups had similar prognoses, even in different $\mathrm{T}$ stages. The exit of mucinous component in gastric adenocarcinoma was not an independent prognostic factor after radical D2 gastrectomy. The results told us that more mucinous component in gastric adenocarcinoma did not lead to worse prognosis after radical D2 gastrectomy, and $\mathrm{N}$ stage and CEA were independent prognostic factors, while the proportion of mucinous component was not.

In our opinion, the tumor microenvironment of gastric adenocarcinoma with mucinous component might play a very important role. Age and sex might affect hormonal levels, especially estrogen. It seems that the mucinous component might secrete some unknown kind of substance to reduce clinical symptoms, which may be the reason more advanced stages were found. Also, alcohol intake for a long time might change the microenvironment. The MGC-specific tumor microenvironment might alter the binding sites of the molecular markers. It is said that the different functions

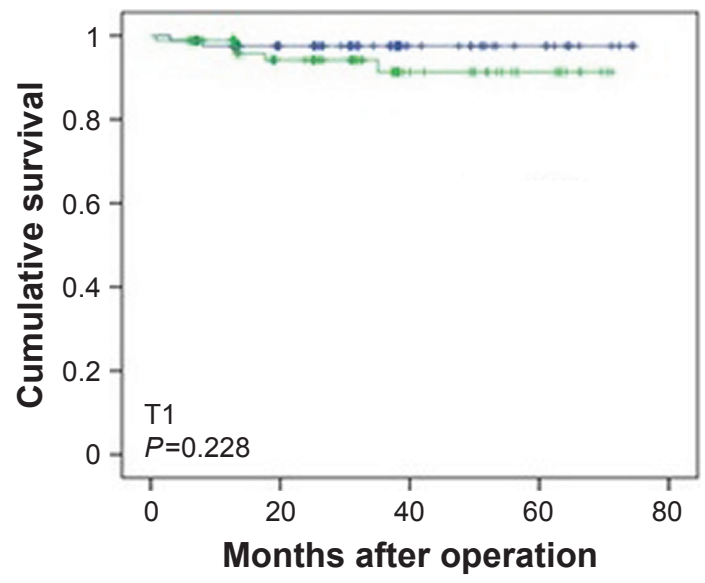

Group
$\sim \mathrm{PGA}+\mathrm{MGC}+\mathrm{PGA}-$ censored + MGC-censored

Figure 2 (Continued) 

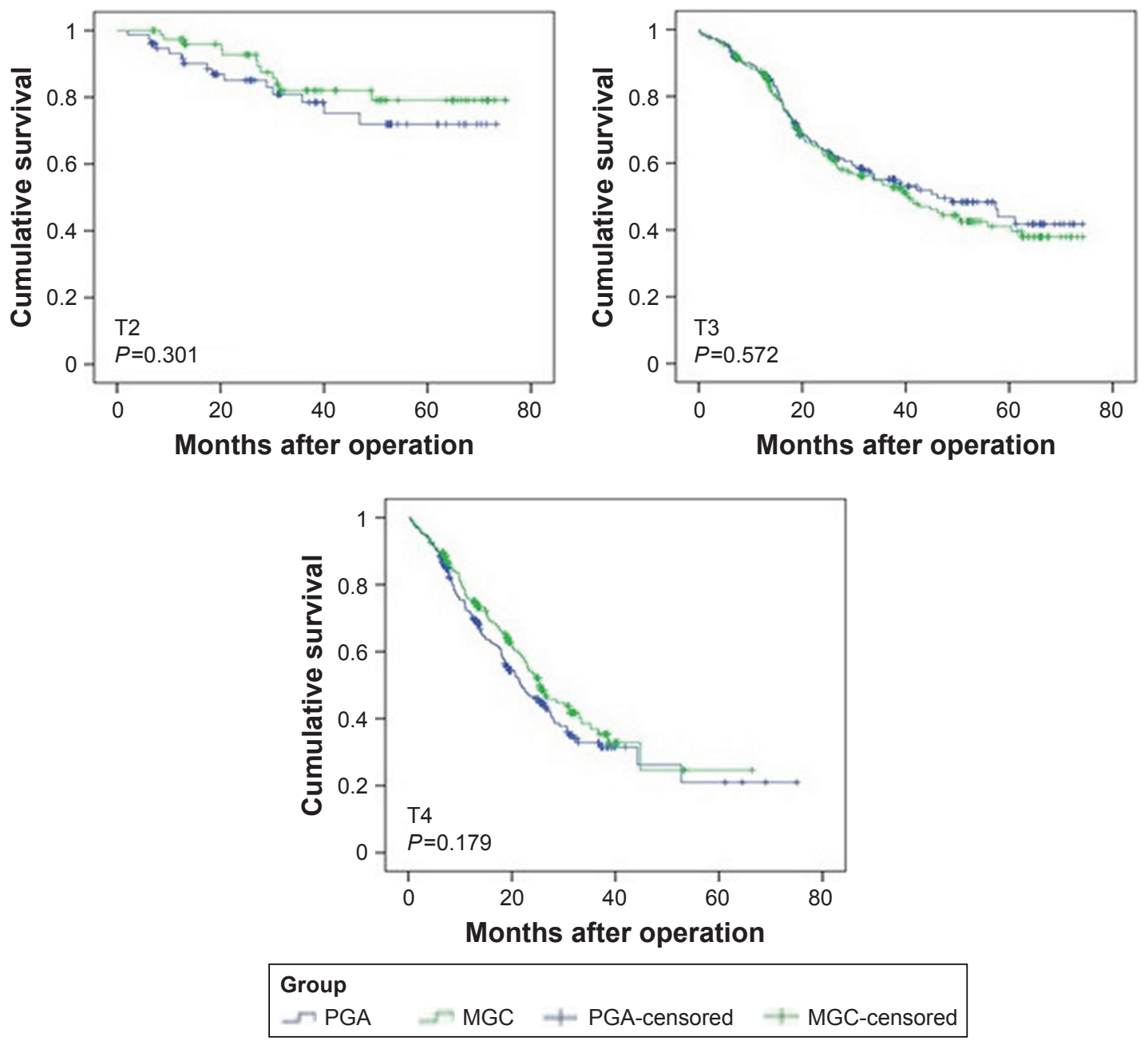

Figure 2 Various proportions of mucinous component showed no difference in OS after radical D2 gastrectomy, even assessed by T stage.

Abbreviations: OS, overall survival; MGC, mucinous gastric carcinoma; GAM, gastric adenocarcinoma with mucinous component; PGA, pure gastric adenocarcinoma.

are caused by different structure, so the higher morbidity of gastric adenocarcinoma in distal gastric might be caused by the enrichment of mucinous cells in glands of this area. However, all these hypotheses need further investigation.

Table 4 Univariate and multivariate analyses of prognostic factors with mucinous component after radical D2 gastrectomy

\begin{tabular}{llll}
\hline Variables & $\beta$ & HR $(95 \% \mathrm{CI})$ & P-value \\
\hline Univariate & & & \\
T stage & 0.758 & $2.134(1.642-2.773)$ & 0 \\
N stage & 0.734 & $2.084(1.722-2.523)$ & 0 \\
Borrmann type & 0.158 & $1.172(1.027-1.337)$ & 0.019 \\
CEA & 0.743 & $2.102(1.454-3.039)$ & 0 \\
AFP & 1.303 & $3.679(1.494-9.056)$ & 0.005 \\
CAI9-9 & 0.773 & $2.167(1.504-3.123)$ & 0 \\
CAI25 & 1.041 & $2.831(1.476-5.429)$ & 0.002 \\
SI00 & 0.934 & $2.543(1.102-5.873)$ & 0.029 \\
Multivariate & & & \\
N stage & 0.518 & $1.679(1.28 I-2.2)$ & 0 \\
CEA & 0.59 & $1.804(1.076-3.025)$ & 0.025 \\
\hline
\end{tabular}

\section{Conclusion}

Whatever its various proportions, mucinous component is not a significant prognosis factor in gastric adenocarcinoma after radical D2 gastrectomy. Specific clinicopathological characteristics and molecular markers with significant differences might provide a potential strategy for optimal individual diagnosis and therapy for gastric adenocarcinoma patients with any proportion of mucinous component. The results of this research should be further confirmed in multicenter studies for future clinical use.

\section{Acknowledgments}

This work was supported by Natural Science Foundation of China grants 81300301 and 81572306 and the Xijing Zhu-Tui Project of the Fourth Military Medical University (XJZT12Z03). Lei Cai, Yan Li and Xue-wen Yang contributed equally to this work and each should be considered first author. 


\section{Author contributions}

All authors made substantial contributions to conception and design, acquisition of data, or analysis and interpretation of data; took part in drafting the article or revising it critically for important intellectual content; gave final approval of the version to be published; and agree to be accountable for all aspects of the work.

\section{Disclosure}

The authors report no conflicts of interest in this work.

\section{References}

1. Kunisaki C, Akiyama H, Nomura M, et al. Clinicopathologic characteristics and surgical outcomes of mucinous gastric carcinoma. Ann Surg Oncol. 2006;13:836-842.

2. Yin C, Li D, Sun Z, et al. Clinicopathologic features and prognosis analysis of mucinous gastric carcinoma. Med Oncol. 2012;29:864-870.

3. Zhang M, Zhu GY, Zhang HF, Gao HY, Han XF, Xue YW. Clinicopathologic characteristics and prognosis of mucinous gastric carcinoma. J Surg Oncol. 2010;102:64-67.

4. Rokutan $\mathrm{H}$, Hosoda F, Hama N, et al. Comprehensive mutation profiling of mucinous gastric carcinoma. J Pathol. 2016;240:137-148.

5. Hsu JT, Wang CW, Le PH, et al. Clinicopathological characteristics and outcomes in stage I-III mucinous gastric adenocarcinoma: a retrospective study at a single medical center. World J Surg Oncol. 2016; $14: 123$.

6. Tang X, Zhang J, Che X, Lan Z, Chen Y, Wang C. The clinicopathological features and long-term survival outcomes of mucinous gastric carcinoma: a consecutive series of 244 cases from a single institute. J Gastrointest Surg. 2016;20:693-699.

7. Isobe T, Hashimoto K, Kizaki J, et al. Characteristics and prognosis of mucinous gastric carcinoma. Mol Clin Oncol. 2015;3:44-50.
8. Zhao J, Ren G, Cai R, et al. Mucinous adenocarcinoma and nonmucinous adenocarcinoma: differing clinicopathological characteristics and computed tomography features in gastric cancer. Oncotarget. 2017;8:45698-45709.

9. Cai L, Li Y, Sun L, et al. Clinicopathologic characteristics and prognosis in patients with mucinous gastric carcinoma after D2 radical gastrectomy. Int J Clin Exp Pathol. 2017;10:2024-2029.

10. Park JY, Ryu MH, Park YS, et al. Prognostic significance of neuroendocrine components in gastric carcinomas. Eur J Cancer. 2014;50: 2802-2809.

11. Chen JH, Cai SR, Wu H. Gastric mucinous cancer histology: clinicopathological characteristics and prognostic value. Gastroenterol Res Pract. 2016;2016:8947505.

12. Niv Y. Helicobacter pylori and gastric mucin expression: a systematic review and meta-analysis. World J Gastroenterol. 2015;21: 9430-9436.

13. Lin X, Zhao Y, Song WM, Zhang B. Molecular classification and prediction in gastric cancer. Comput Struct Biotechnol J. 2015;13:448-458.

14. Boltin D, Niv Y. Mucins in gastric cancer: an update. $J$ Gastrointest Dig Syst. 2013;3:15519.

15. Cai L, Li Y, Yang XW, et al. Is the signet ring cell histological type a positive prognostic factor for gastric adenocarcinoma after D2 radical gastrectomy? Int J Clin Exp Pathol. 2017;10:10489-10494.

16. Zu H, Wang H, Li C, Xue Y. Clinicopathologic characteristics and prognostic value of various histological types in advanced gastric cancer. Int J Clin Exp Pathol. 2014;7:5692-5700.

17. Jiang H, Zhang H, Tian L, Zhang X, Xue Y. The difference in clinicpathological features between signet ring cell carcinoma and gastric mucinous adenocarcinoma. Tumour Biol. 2013;34:2625-2631.

18. Oue N, Sentani K, Sakamoto N, Yasui W. Clinicopathologic and molecular characteristics of gastric cancer showing gastric and intestinal mucin phenotype. Cancer Sci. 2015;106:951-958.

19. Kim KH, Kim MC, Jung GJ, Kim SJ. The differences in clinicopathological features and prognosis among the subtypes of signet ring cell, mucinous, papillary, and lymphoepithelioma-like carcinoma in advanced gastric cancer. Hepatogastroenterology. 2014;61: 2149-2155.
OncoTargets and Therapy

\section{Publish your work in this journal}

OncoTargets and Therapy is an international, peer-reviewed, open access journal focusing on the pathological basis of all cancers, potential targets for therapy and treatment protocols employed to improve the management of cancer patients. The journal also focuses on the impact of management programs and new therapeutic agents and protocols on

\section{Dovepress}

patient perspectives such as quality of life, adherence and satisfaction. The manuscript management system is completely online and includes a very quick and fair peer-review system, which is all easy to use. Visit http://www.dovepress.com/testimonials.php to read real quotes from published authors. 